\title{
ESPECTROFOTOMETRIA DERIVATIVA: UMA ESTRATÉGIA SIMPLES PARA A DETERMINAÇÃO SIMULTÂNEA DE CORANTES EM ALIMENTOS
}

\author{
Eliane Cristina Vidotti e Maria do Carmo E. Rollemberg* \\ Departamento de Química, Universidade Estadual de Maringá, Av. Colombo, 5790, 87020-900 Maringá - PR
}

Recebido em 17/1/05; aceito em 22/7/05; publicado na web em 13/1/06

\begin{abstract}
DERIVATIVE SPECTROPHOTOMETRY: A SIMPLE STRATEGY FOR SIMULTANEOUS DETERMINATION OF FOOD DYES. A very simple spectrophotometric method is described for resolving binary mixture of the food colorants Sunset Yellow (INS 110) and Tartrazine Yellow (INS 102) by using the first derivative spectra with measurements at zero-crossing wavelengths. Before the spectrophotometric measurements, the dyes were sorbed onto polyurethane foam and recovered in $N, N$-dimethilformamide. Commercial food products (gelatine and juice powder) were analysed by using the proposed method and the HPLC technique. The results are in very good agreement and the differences between the methods is not statistically important. Therefore, the first-order derivative spectrophotometric method is accurate, precise, reliable and could be applied to the routine analysis of food samples.
\end{abstract}

Keywords: first derivative spectrophotometry; polyurethane foam; food dyes.

\section{INTRODUÇÃO}

Muitos produtos alimentícios originalmente não apresentam cor, e em outros a cor é perdida ou alterada durante o processo de industrialização; assim, surge a necessidade da adição de corantes a fim de conferir ou restaurar a coloração perdida ${ }^{1}$.

Os corantes amarelo crepúsculo e amarelo tartrazina são corantes sintéticos que apresentam estruturas distintas das substâncias corantes encontradas na natureza (Figura 1). Estes corantes são comumente adicionados aos alimentos, proporcionando coloração intensa e estável ao produto industrializado. Assim como para muitos outros aditivos alimentares, o controle analítico dos corantes sintéticos é de grande importância na indústria alimentícia, devido ao potencial tóxico e carcinogênico que apresentam ${ }^{2-4}$.

a)

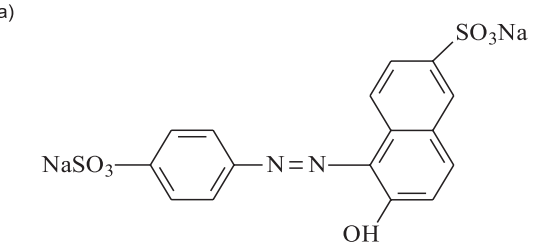

b)

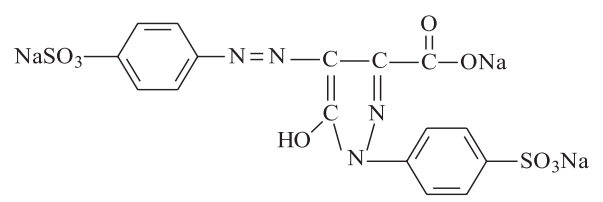

Figura 1. Estruturas dos corantes: (a) amarelo crepúsculo e (b) amarelo tartrazina

Muitos métodos analíticos têm sido descritos para determinar misturas de corantes em alimentos, incluindo cromatografia ${ }^{5}$, espectrofotometria ${ }^{6-8}$, voltametria ${ }^{9-11}$, eletroforese capilar $^{12}$ e várias combinações de técnicas ${ }^{13,14}$. Os métodos baseados no uso

*e-mail: mcerollemberg@uem.br da espectrofotometria derivativa têm apresentado resultados satisfatórios na determinação de misturas de corantes em alimentos ${ }^{15,16}$.

A espectrofotometria derivativa é, atualmente uma ferramenta analítica adicional que auxilia na resolução de diversos problemas analíticos. A derivatização dos espectros permite separar sinais sobrepostos e eliminar "background" causado pela presença de outras espécies na amostra. Portanto, torna-se desnecessária uma etapa preliminar de separação das espécies a serem determinadas, simplificando o procedimento analítico e aumentando a velocidade analítica ${ }^{17,18}$.

A espectrofotometria derivativa consiste na representação da razão da variação da absorvância com o comprimento de onda, em função do comprimento de onda. A diferenciação da lei de LambertBeer $\left(d^{n} A / d \lambda^{n}=\operatorname{cb}\left(d^{n} \varepsilon / d \lambda^{n}\right)\right)$ mostra que as derivadas são sempre proporcionais às concentrações do analito, sendo as aplicações analíticas baseadas nesta relação ${ }^{19}$.

O objetivo do presente trabalho é determinar simultaneamente os corantes amarelo crepúsculo e amarelo tartrazina em produtos alimentícios, utilizando o método espectrofotométrico derivativo. Para minimizar possíveis efeitos de interferências da matriz os corantes foram inicialmente retidos em espuma de poliuretana, e recuperados usando o solvente orgânico $N, N$-dimetilformamida.

\section{PARTE EXPERIMENTAL}

\section{Reagentes}

Os corantes amarelo crepúsculo (INS 110) e amarelo tartrazina (INS 102) foram adquiridos da indústria Duas Rodas (Santa Catarina, Brasil) e purificados, através de sucessivos processos de extrações com solventes orgânicos; após a etapa de purificação, os corantes foram secos em estufa por $12 \mathrm{~h}$ à temperatura de $60^{\circ} \mathrm{C}$. $\mathrm{O}$ grau de pureza foi determinado por comparação a dados da literatura $^{1}$, sendo obtidos 89 e $91 \%$ para amarelo crepúsculo e amarelo tartrazina, respectivamente. A solução estoque de concentração $1000 \mathrm{mg} / \mathrm{L}$ foi preparada pela dissolução em água destilada da massa apropriada de cada corante. Utilizou-se solução tampão acetato $(\mathrm{pH}$ $3,0)$ com concentração de $1,0 \mathrm{~mol} / \mathrm{L}$ para ajuste das condições de 
retenção dos corantes na espuma de poliuretana. Todos os solventes e reagentes utilizados foram de grau analítico. A espuma de poliuretana do tipo poliéster $\left(\mathrm{d}=60 \mathrm{~kg} / \mathrm{m}^{3}\right)$ foi lavada com água e acetona, seca e triturada $(600 \mu \mathrm{m})$ antes do uso.

\section{Procedimento para determinação dos corantes}

Uma alíquota de 10,0 mL da solução (amostra ou padrão) foi transferida para um balão de $25,0 \mathrm{~mL}$ e a esse volume foi adicionado um volume igual de $\mathrm{CH}_{3} \mathrm{COOH}(2,0 \mathrm{~mol} / \mathrm{L})$, sendo o $\mathrm{pH}$ ajustado a 3,0 com $\mathrm{NaOH}(1,0 \mathrm{~mol} / \mathrm{L})$. A mistura de $150 \mathrm{mg}$ de espuma de poliuretana (EPU) e um volume de $10,0 \mathrm{~mL}$ da solução tamponada foi agitada (agitador magnético) por um período de $20 \mathrm{~min}$. Em seguida a mistura foi filtrada e a espuma seca por $30 \mathrm{~min}$, à temperatura de $60^{\circ} \mathrm{C}$. Após a secagem os corantes foram recuperados utilizando 20,0 $\mathrm{mL}$ de $N, N$-dimetilformamida, sob agitação (agitador magnético) por $20 \mathrm{~min}$. Os corantes foram determinados como descritos a seguir. A solução do branco contendo todos os reagentes exceto o analito e as soluções padrão foram preparadas e tratadas segundo o mesmo procedimento descrito para as amostras.

\section{Preparação das amostras de alimentos}

$\mathrm{O}$ método foi aplicado à determinação dos corantes amarelo crepúsculo e amarelo tartrazina em alguns produtos alimentícios: pós para gelatina (sabores laranja e maracujá): foram dissolvidos em água quente $\left(\sim 60^{\circ} \mathrm{C}\right)$ de acordo com as recomendações do fabricante e filtrados em membrana $0,45 \mu \mathrm{m}$ e, pós para refresco (sabores laranja, manga e pêssego): foram dissolvidos em água, à temperatura ambiente, de acordo com as recomendações do fabricante e filtrados em membrana $0,45 \mu \mathrm{m}$.

\section{Aplicação da técnica da primeira derivada}

Os espectros de absorção das amostras e das misturas binárias dos corantes em quantidades conhecidas (padrões com concentrações na faixa entre 1-20 mg/L para cada corante) foram obtidos na região entre 350 e $700 \mathrm{~nm}$, com velocidade de varredura de 350 $\mathrm{nm} / \mathrm{min}$ (Hitachi U-2000 Spectrophotometer). O espectro da primeira derivada foi obtido com $\Delta \lambda=3 \mathrm{~nm}$ e aplicando sobre 11 pontos experimentais a função "smoothing", usando o método simplificado de Savitzky-Golay ${ }^{20}$. Os sinais no ponto zero dos espectros de absorção das misturas binárias foram medidos usando curvas de trabalho apropriadas. Estas curvas de trabalho foram construídas a partir da medida do sinal analítico de soluções com diferentes proporções dos corantes nas soluções.

\section{Procedimento cromatográfico}

A comparação do método espectrofotométrico derivativo foi realizada com a determinação dos corantes amarelo crepúsculo e amarelo tartrazina nos produtos alimentícios através da CLAE, usando um detector de arranjo de diodos com medidas em $252 \mathrm{~nm}$ (absorvância máxima para os corantes analisados). Como fase estacionária foi utilizada uma coluna cromatográfica $\mathrm{C}_{18}$ e como fase móvel duas diferentes soluções: tampão de $\mathrm{NaH}_{2} \mathrm{PO}_{4} / \mathrm{Na}_{2} \mathrm{HPO}_{4}$ $0,01 \mathrm{~mol} / \mathrm{L}(90 \% \mathrm{v} / \mathrm{v})$ e metanol $(10 \% \mathrm{v} / \mathrm{v}), \mathrm{pH} 7,0$ (solução A) e tampão $\mathrm{NaH}_{2} \mathrm{PO}_{4} / \mathrm{Na}_{2} \mathrm{HPO}_{4} 0,01 \mathrm{~mol} / \mathrm{L}(20 \% \mathrm{v} / \mathrm{v})$ e metanol $(80 \%$ v/v), pH 7,0 (solução B).

O gradiente de eluição foi conduzido de $100 \%$ da solução A até $100 \%$ da solução B em um intervalo de $40 \mathrm{~min}$, com vazão de 1,0 mL/ min. Os tempos de retenção obtidos foram 10,0 e 17,4 min para os corantes tartrazina e amarelo crepúsculo, respectivamente ${ }^{21-23}$.

\section{RESULTADOS E DISCUSSÃO}

Os corantes amarelo crepúsculo e amarelo tartrazina são espécies com absorção espectral na região do visível e em misturas uma intensa sobreposição espectral das bandas de absorção impede a aplicação da espectrofotometria direta para análises quantitativas (Figura 2). Em situações como esta, as técnicas derivativas têm sido aplicadas com sucesso para melhorar a sensibilidade e a seletividade das determinações.

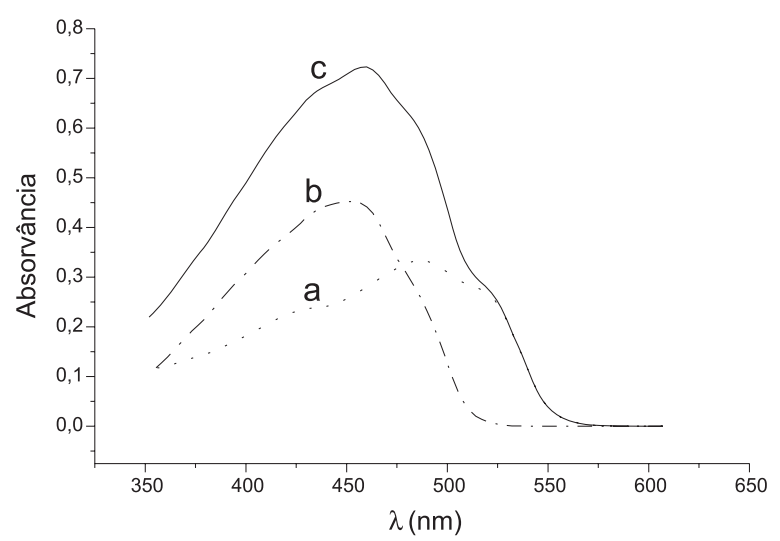

Figura 2. Espectros de absorção em DMF para os corantes amarelo crepúsculo (a, 10,0 mg/L), amarelo tartrazina (b, 10,0 mg/L) e mistura (c)

O problema na determinação quantitativa dos corantes sintéticos não é apenas a separação dos mesmos, mas isolar quantitativamente os corantes da matriz. Isto é muitas vezes necessário devido à complexidade da matriz, capaz de causar sérias interferências nas medidas espectrofotométricas, gerando erros na medida analítica.

Métodos tradicionais, semelhantes à absorção em algodão ou poliamidas, não parecem ser quantitativos e podem ainda resultar na degradação dos corantes ${ }^{24}$.

Nos últimos anos, a utilização da espuma de poliuretana vem se destacando devido aos bons resultados apresentados em procedimentos de separação/pré-concentração de espécies orgânicas e inorgânicas ${ }^{25-27}$. Neste trabalho, foi explorada a propriedade de sorção apresentada pela espuma de poliuretana, com objetivo de isolar os corantes da matriz, eliminando eventuais problemas na medida espectrofotométrica.

Estudos sugerem que a adsorção dos corantes na espuma de poliuretana ocorre quando a espécie (corante) predominante está em sua forma neutra (zwitterion), sendo a adsorção de cada espécie uma combinação de efeitos estéricos, indutivos e de natureza hidrofóbica da espécie ${ }^{28}$. O processo de sorção dos corantes na espuma de poliuretana é afetado por diferentes fatores, como $\mathrm{pH}$, quantidade de EPU e tempo de contato. Cada uma dessas variáveis foi otimizada, sendo definidas as condições: $150 \mathrm{mg}$ de EPU, $20 \mathrm{~min}$ de agitação e solução tampão acetato $1,0 \mathrm{~mol} / \mathrm{L}$ (pH 3,0). De acordo com os valores de pKa para os corantes estudados ${ }^{29}$, em $\mathrm{pH} \mathrm{3,0}$ o grupo sulfônico está não protonado e, com a protonação do grupo azo, a molécula está na forma neutra, condição essa favorável à retenção $0^{28}$.

Diferentes solventes foram testados a fim de recuperar os corantes retidos na espuma de poliuretana. A recuperação dos corantes em solução aquosa alcalina mostrou-se pouco eficiente, sendo necessário um longo tempo de contato, o que poderia contribuir para degradação dos corantes devido ao meio alcalino.

O solvente orgânico $N, N$-dimetilformamida apresentou resultados satisfatórios para uma recuperação eficiente dos corantes. A 
eficiência na recuperação dos corantes utilizando DMF foi 98-101\% e $97-100 \%$ para amarelo crepúsculo e amarelo tartrazina, respectivamente. Uma hipótese sugerida para o favorecimento da dessorção dos corantes nesse meio é a solvatação, pelo solvente aprótico apolar $N, N$-dimetilformamida ${ }^{30}$.

A recuperação foi possível agitando-se a espuma, contendo os corantes retidos, durante $10 \mathrm{~min}$ e utilizando $20,0 \mathrm{~mL}$ do solvente, após a etapa de secagem, à temperatura de $60^{\circ} \mathrm{C}$. O estudo do comportamento termogravimétrico dos corantes indicou que a temperatura utilizada para secagem não afeta a estabilidade dos corantes de interesse.

Para alcançar melhores precisão e exatidão no método, foi necessário um estudo dos parâmetros instrumentais que afetam a forma da derivada espectral: a velocidade de varredura, a aplicação da função de atenuação "smoothing" e o incremento do comprimento de onda para obter a derivada espectral $(\Delta \lambda)$. Sinais analíticos semelhantes foram obtidos para diferentes velocidades de varreduras, sendo desta forma escolhido um valor intermediário, $350 \mathrm{~nm} / \mathrm{min}$. Para a mistura binária estudada (mistura dos corantes amarelo crepúsculo e tartrazina) um $\Delta \lambda=3 \mathrm{~nm}$ foi selecionado como o melhor, considerando o nível de ruído, a resolução no espectro e a concentração do analito. Devido ao grande nível de ruído, foi necessário aplicar a função "smoothing" considerando 11 pontos experimentais.

De acordo com o método proposto, para analisar misturas binárias é preciso medir o sinal no comprimento de onda em que a contribuição de um dos componentes da mistura seja zero. Estes comprimentos de onda foram previamente estabelecidos para cada um dos corantes.

As curvas da primeira derivada dos espectros obtidos para amarelo crepúsculo e amarelo tartrazina puros e para a mistura destes corantes estão apresentadas na Figura 3, sendo possível observar os comprimentos de onda selecionados: $550,0 \mathrm{~nm}$ (sinal zero para tartrazina) e 486,5 nm (sinal zero para crepúsculo) para determinação dos corantes amarelo crepúsculo e tartrazina, respectivamente.

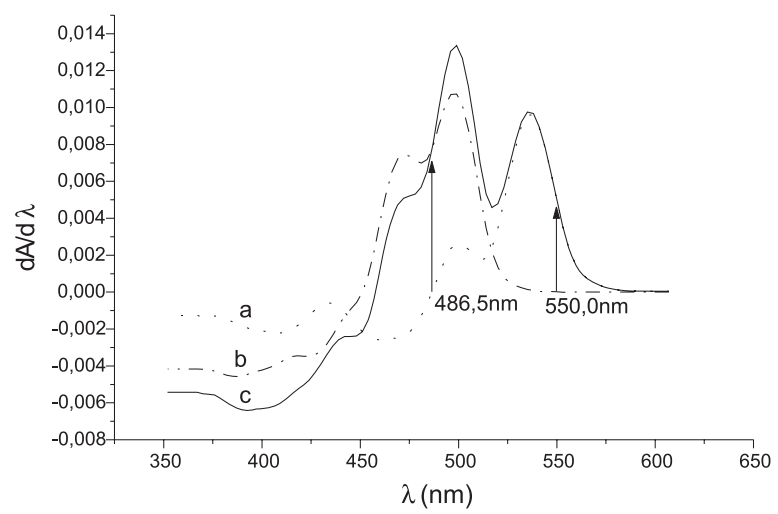

Figura 3. Espectro da primeira derivada para os corantes amarelo crepúsculo (a, 10,0 mg/L), amarelo tartrazina (b, 10,0 mg/L) e mistura (c). Tartrazina foi determinado em 486,5 $\mathrm{nm}$ e crepúsculo foi determinado em 550,0 nm

As curvas de trabalho foram obtidas nos comprimentos de onda selecionados para misturas sintéticas binárias, apresentando linearidade na faixa de concentração de $1-20 \mathrm{mg} / \mathrm{L}$. A Figura 4 apresenta os espectros derivados para diferentes concentrações dos corantes, mostrando a proporcionalidade entre as derivadas $\mathrm{d}^{\mathrm{n}} \mathrm{A} /$ $\mathrm{d} \lambda^{\mathrm{n}}$ e as concentrações dos analitos.

O limite de detecção foi determinado para cada corante considerando a mistura binária de interesse, a partir de 10 medidas do branco do procedimento, obtendo-se a média e o desvio padrão desse conjunto de medidas. Os limites de detecção $\left(3 \mathrm{~S}_{\mathrm{B}} / \mathrm{m}\right.$, sendo

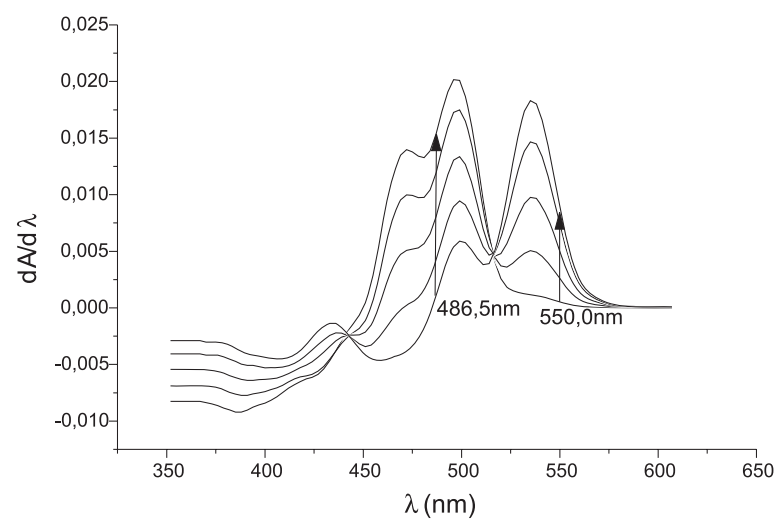

Figura 4. Espectros da sobreposição espectral da mistura entre os corantes amarelo crepúsculo e amarelo tartrazina em diferentes concentrações $(1-20 \mathrm{mg} / \mathrm{L})$

$\mathrm{S}_{\mathrm{B}}$ o desvio padrão das medidas do branco do procedimento e $\mathrm{m}$ a inclinação da curva de trabalho) estimados para os corantes foram 0,38 e $0,19 \mathrm{mg} / \mathrm{L}$ para amarelo crepúsculo e amarelo tartrazina respectivamente. De modo semelhante, estimou-se o limite de quantificação para os corantes de interesse, sendo esses valores 1,26 para amarelo crepúsculo e $0,63 \mathrm{mg} / \mathrm{L}$ para amarelo tartrazina ${ }^{30}$.

Foram estabelecidas curvas de trabalho com coeficientes de correlação $\left(\mathrm{r}^{2}\right)$ superiores a 0,999 no intervalo de concentrações de 1,0-20,0 $\mathrm{mg} / \mathrm{L}$ para os corantes analisados.

O método foi aplicado à análise de misturas binárias sintéticas dos corantes investigados, com recuperações superiores a $97 \%$ (97-101\%).

Foram realizadas medidas independentes para avaliar a precisão do método. Resultados satisfatórios foram obtidos para misturas contendo concentrações iguais a $2,0 \mathrm{mg} / \mathrm{L}$ de amarelo crepúsculo e amarelo tartrazina. Nessas condições, a precisão (\%) mostrou-se melhor que $2 \%$.

As amostras de pós para gelatina e de pós para suco foram preparadas como descritos na seção experimental, e as soluções obtidas foram analisadas através do método espectrofotométrico derivativo proposto. $\mathrm{O}$ estudo da recuperação dos corantes em uma amostra de suco e de gelatina foi realizado pela adição de quantidades exatas de cada corante às amostras, antes da retenção na EPU. A Tabela 1 mostra que a recuperação para amarelo crepúsculo e amarelo tartrazina foi bastante satisfatória. A recuperação média, desvio padrão e desvio padrão relativo foram de 97,80, 1,97 e $2,01 \%$ para amarelo crepúsculo e $97,14,1,71$ e $1,76 \%$ para amarelo tartrazina.

Mesmo com a etapa preliminar de retenção na EPU, o procedimento proposto ainda é comparável a outros descritos na literatura, envolvendo técnicas cromatográficas, quanto à velocidade analítica. Por outro lado, a simplicidade e facilidade de execução tornam este procedimento espectrofotométrico proposto adequado às determinações de rotina.

A análise estatística (teste t de Student) dos resultados obtidos pelos dois métodos, para um nível de confiança de $95 \%$ mostrou não haver diferenças significativas entre os métodos; os valores de t de Student calculados $(0,346$ - 4,041) mostraram-se inferiores ao valor teórico $(4,303)^{31}$.

Os resultados analíticos obtidos nas determinações dos corantes analisados no presente trabalho são aceitáveis pela legislação brasileira, que permite a utilização de $0,01 \%$ de corante na massa final do produto a ser consumido ${ }^{32}$.

Como a etapa de retenção na EPU livra os corantes da matriz, eliminando possíveis interferências nas medidas espectrofotomé- 
Tabela 1. Determinação e recuperação dos corantes artificiais amarelo crepúsculo e amarelo tartrazina em produtos alimentícios"

\begin{tabular}{|c|c|c|c|c|c|c|}
\hline amostras & presente $(\mathrm{mg} / \mathrm{L})$ & $\begin{array}{l}\text { amarelo crepúsculo } \\
\text { adicionado }(\mathrm{mg} / \mathrm{L})\end{array}$ & recuperado $(\%)$ & \multicolumn{3}{|c|}{ amarelo tartrazina } \\
\hline pó para gelatina & 1,76 & 1,00 & 100,7 & 1,50 & 3,00 & 95,4 \\
\hline (sabor pêssego) & 1,76 & 3,00 & 99,3 & 1,50 & 1,00 & 99,5 \\
\hline pó para suco & 3,14 & 2,00 & 96,5 & 4,08 & 2,00 & 98,7 \\
\hline \multirow[t]{2}{*}{ (frutas cítricas) } & 3,14 & 3,00 & 95,2 & 4,08 & 1,00 & 95,3 \\
\hline & 3,14 & 1,00 & 97,3 & 4,08 & 2,00 & 96,8 \\
\hline valores médios & & & 97,80 & & & 97,14 \\
\hline desvio padrão & & & 1,97 & & & 1,71 \\
\hline desvio padrão rel & $(\%)$ & & 2,01 & & & 1,76 \\
\hline
\end{tabular}

*valores médios de três determinações.

Tabela 2. Determinação dos corantes artificiais amarelo crepúsculo e amarelo tartrazina em produtos alimentícios através da espectrofotometria derivativa e cromatografia líquida de alta eficiência (CLAE)*

\begin{tabular}{lcccc}
\hline Produtos alimentícios & \multicolumn{2}{c}{$\begin{array}{c}\text { Espectrofotometria Derivativa (mg/g) } \\
\text { trepúsculo }\end{array}$} & \multicolumn{2}{c}{ CLAE (mg/g) } \\
& tartrazina & \multicolumn{2}{c}{ crepúsculo } & tartrazina \\
\hline pó para gelatina (sabor laranja) & $0,057 \pm 0,009$ & $0,056 \pm 0,002$ & $0,059 \pm 0,014$ & $0,057 \pm 0,001$ \\
pó para gelatina (sabor maracujá) & $0,028 \pm 0,002$ & $0,178 \pm 0,002$ & $0,030 \pm 0,001$ & $0,182 \pm 0,008$ \\
pó para suco (sabor laranja) & $0,488 \pm 0,003$ & $0,618 \pm 0,009$ & $0,495 \pm 0,016$ & $0,622 \pm 0,004$ \\
pó para suco (sabor pêssego) & $0,108 \pm 0,010$ & $0,148 \pm 0,002$ & $0,110 \pm 0,001$ & $0,148 \pm 0,003$ \\
pó para suco(sabor manga) & $1,175 \pm 0,018$ & $1,210 \pm 0,001$ & $1,201 \pm 0,019$ & $1,212 \pm 0,028$ \\
\hline
\end{tabular}

*valores médios de três determinações e desvio padrão

tricas, o método pode ser aplicado a outros produtos alimentícios de composição mais complexa, tais como bolachas (recheios) doces, balas, doces industrializados ou pós para sorvetes (dados não publicados).

\section{CONCLUSÕES}

O método espectrofotométrico derivativo proposto foi aplicado com sucesso a amostras de pós para gelatina e pós para suco.

$\mathrm{Na}$ literatura não há método oficial para se investigar o teor em corantes sintéticos nos alimentos. Então, para validar o procedimento proposto recorreu-se a um método independente, a cromatografia líquida de alta eficiência. Uma comparação entre os valores obtidos nas análises de alguns produtos pelo método espectrofotométrico e pela CLAE está apresentada na Tabela 2.

$\mathrm{O}$ método proposto permite a determinação simples, rápida e direta dos corantes amarelo crepúsculo e amarelo tartrazina em alimentos, sem separações prévias; além disso, o procedimento espectrofotométrico é de baixo custo. $\mathrm{O}$ estudo da recuperação indica que o procedimento proposto é atraente para determinações de rotina.

Na comparação do método espectrofotométrico derivativo proposto, a CLAE, a concordância entre os valores obtidos mostra a confiabilidade do método proposto para resolução de sobreposições espectrais sem interferências da matriz.

\section{AGRADECIMENTOS}

À CAPES pela bolsa de doutorado concedida à E. C. Vidotti.

\section{REFERÊNCIAS}

1. Takahashi, M. Y.; Yabiku, H. Y.; Marsiglia, D. A. P.; Rev. Inst. Adolfo Lutz 1988, 48, 07.

2. Altinöz, S.; Toptan, S.; J. Food Comp. Anal. 2003, 16, 517.

3. Combes, R. D.; Haveland-Smith, R. B.; Mutat. Res. 1982, 98, 101.

4. Machinski, M. Jr.; Food Addit. Contam. 1998, 15, 481.
5. Gennaro, M. C.; Gionnini, E.; Angelino, S.; Aigotti, R.; Giacosa, D.; J. Chromatogr., A 1997, 767, 87.

6. Berzas-Nevado, J. J.; Guiberteau-Cabanillas, C.; Contento-Salcedo, A. M.; Martin-Villamiulas, R.; Quim. Anal. 2001, 20, 63.

7. Slampova, A.; Smela, D.; Vandrackova, A.; Jancarova, I.; Kuba, V.; Chem. Listy. 2001, 95, 163.

8. Ozgur, M. U.; Alpdogan, G.; Koyncu, I.; Anal. Lett. 2002, 35, 721.

9. Becerro Dominguez, F.; Gonzales Diego, F.; Hernandez Mendez, J.; Talanta 1990, 37, 655 .

10. Lopez-de-Alba, P. L.; Lopez-Martinez, L.; De-Leon-Rodriguez, L. M.; Electroanal. 2002, 14, 197.

11. Berzas Nevado, J. J.; Rodriguez Flores, J.; Villasenõr Llerena, M. J.; Talanta 1997, 44, 467.

12. Del Giovine, L.; Piccioli Bocca, A.; Food Control. 2003, 14, 131.

13. Fush, M. -R.; Chia, K. -J.; Talanta 2002, 56, 663.

14. Greenway, G. M.; Kometa, N.; Macroe, R.; Food Chem. 1992, 43, 137.

15. Berzas Nevado, J. J.; Rodriguez Flores, J.; Villasenõr Llerena, M. J.; Talanta 1993, 40, 1391.

16. Ni, Y.; Gong, X.; Anal. Chim. Acta 1997, 354, 163.

17. Sayar, S.; Özdemir, Y.; Food Chem. 1998, 61, 367.

18. Berzas-Nevado, J. J.; Guiberteau-Cabanillas, C.; Contento-Salcedo, A. M.; Talanta 1995, 42, 2043.

19. Rocha, F. R. P.; Teixeira, L. S. G.; Quim. Nova 2004, 27, 807.

20. Savitzky, A.; Golay, M. J.; Anal. Chem. 1964, 36, 1627.

21. Berzas Nevado, J.; Rodríguez Florez, J.; Guiberteau-Cabanillas, C.; Llerena, M. J. V.; Contendo-Salcedo, A.; Talanta 1998, 46, 933.

22. Berzas Nevado, J.; Guiberteau-Cabanillas, C.; Contendo-Salcedo, A.; Anal. Lett. 1998, 31, 2513.

23. www.waters.com/watersDivision/pdfs/catalog/Eappnotebook/ compod_index_C.htm, acessada em Outubro 2004.

24. Rzeszntek, K.; Chow, A.; Talanta 1998, 46, 507.

25. Ferreira, S. L. C.; Santos, H. C.; Campos, R. C.; Talanta 2003, 61, 789

26. El-Shahawi, M. S.; Nassif, H. A.; Anal. Chim. Acta 2003, 487, 249.

27. Werbowesky, R.; Chow, A.; Talanta 1996, 43, 263.

28. Vidotti, E. C.; Cancino, J. C.; Oliveira, C. C.; Rollemberg, M. C. E.; Anal. Sci. 2005, 21, 149.

29. Das, R.; Mitra, S.; Mukherjee, S.; J. Photochem. Photobiol., A 1993, 76, 33.

30. Altinöz, S.; Toptan, S.; J. Food Comp. Anal. 2002, 15, 667.

31. Harris, D. C.; Análise Química Quantitativa, 5a ed., Rio de Janeiro, 2001, cap. 4.

32. Brasil. Leis, decretos etc.- Resolução normativa $n^{\circ} 4 / 78$ da Câmara de Alimentos do Conselho Nacional de Saúde. Diário Oficial, Brasília, 2 out. 1978, Seção I, p. 15944. 